\title{
Comparison of different methods to rectify IKONOS imagery without use of sensor viewing geometry
}

\author{
Oscar Rosario Belfiore, Claudio Parente \\ Department of Sciences and Technologies, University of Naples "Parthenope", Naples, Italy \\ Email address: \\ oscarrosario.belfiore@uniparthenope.it (O. R. Belfiore), claudio.parente@uniparthenope.it (C. Parente)
}

\section{To cite this article:}

Oscar Rosario Belfiore, Claudio Parente. Comparison of Different Methods to Rectify IKONOS Imagery without Use of Sensor Viewing Geometry. American Journal of Remote Sensing. Vol. 2, No. 3, 2014, pp. 15-19. doi: 10.11648/j.ajrs.20140203.11

\begin{abstract}
Satellite images can be rectified and adapted to map representation also without information about viewing geometry of the sensor. Polynomial Functions (PFs) or Rational Polynomial Functions (RPFs) can be applied for this purpose, both requiring Ground Control Points (GCPs), of which the positions in the image as well as in the real world must be known. Typically for PFs only planimetric (X, Y) positions of GCPs are used while for RPFs 3D coordinates (X, Y, Z) of them as well as a DEM (Digital Elevation Model) of the entire scene are required. Check Points (CPs) with the same characteristics of GCPs (but not coincident with them) are used to better verify the accuracy of the product. Not only topographic survey, but also maps or ortho-photos with adequate resolution supply the coordinates of GCPs as well as CPs. This paper is aimed to compare methods to rectify IKONOS images based on PFs or RPFs applications, considering the positional accuracy of the results as index for performance evaluation. Tests were executed on IKONOS panchromatic image of an area of the Cilento and Vallo di Diano National Park, in Campania Region (Italy): ortho-photos (scale 1:10,000) were used for GCPs and CPs planimetric position in the real world while for RPFs applications also DEM (cell size $20 \mathrm{~m}$ ) was considered as source of 3D information. To compare the selected methods, differences (residuals) between the X, Y coordinates of GCPs (but also of the CPs) on the ortho-photos and corresponding values in the rectified image were calculated and evaluated. The positional accuracy of the resulting products in relation to the method as well as to the number of GCPs was analyzed; also the implications of the calculation of Rational Polynomial Coefficients (RPCs) in alternative to the use of the values supplied for them by the image provider were investigated.
\end{abstract}

Keywords: IKONOS Satellite Imagery, Rectification, Polynomial Functions, Rational Polynomial Functions, Rational Polynomial Coefficients

\section{Introduction}

IKONOS is the world's first high-resolution commercially-available satellite. Launched into space on September 24, 1999 from a pad at Vandenberg Air Force Base, CA, IKONOS was originally owned by Space Imaging and is now a part of GeoEye's constellation of high resolution satellites [1]. Two types of sensors are present on board of IKONOS satellite: the first collects panchromatic images $(0.526 \mu \mathrm{m}-0.929 \mu \mathrm{m})$ with 0.82 meter resolution at nadir, the second multispectral ones (Blue: $0.445 \mu \mathrm{m}-0.516 \mu \mathrm{m}$; Green: $0.506 \mu \mathrm{m}-0.595 \mu \mathrm{m}$; Red: $0.632 \mu \mathrm{m}-0.698 \mu \mathrm{m}$; Near Infrared: $0.757 \mu \mathrm{m}-0.853 \mu \mathrm{m}$ ) with 3.28 meter resolution at nadir. Usually commercial products present cell sizes down-resampled to $1 \mathrm{~m}$ for panchromatic images, $4 \mathrm{~m}$ for multispectral ones [2].

If geometrically corrected and georeferenced, IKONOS images can be used for many purposes, such as map creation and updating, change detection and so on. In fact they supply detailed information about morphological configurations, urban environments [3], land cover and use, forests, waters (sea, lake, river) and other landscape elements [4].

Thanks to their high level of spatial and spectral information, IKONOS images are also used to establish the variation of shorelines in presence of coastal erosion [5]. Of course positional accuracy occurs, so particular attention must be reserved to geometrical correction and georeferencing process; these operations are usually made at the same time adapting the image to the cartographic plane with geodetic coordinates. In this paper rectification is conducted on IKONOS panchromatic image using methods that are alternative to rigorous approach based on sensor characteristics and acquisition mode. Positional accuracies supplied by the different considered methods are compared and discussed. 


\section{Methods for Satellite Images Rectification}

Because of their significant geometric distortions, satellite raw images cannot be directly used: overlay with maps and other GIS layers is possible only if they are transformed and adapted to the selected cartographic projection. To rectify low and medium resolution satellite images Polynomial Functions (PFS) are usually applied [6].

Typically 2D models are used, so images coordinates ( $\left.\mathrm{X}^{\prime}, \mathrm{Y}^{\prime}\right)$ and cartographic ones $(\mathrm{X}, \mathrm{Y})$ are related by the following equations:

$$
X^{\prime}=P_{1}^{n}(X, Y) \quad Y^{\prime}=P_{2}^{n}(X, Y)
$$

where $P_{l}^{n}$ is the generic polynomial ( $n$ is the order) represented by:

$$
P_{l}^{n}(X, Y)=\sum_{i=0}^{m_{1}} \sum_{j=0}^{m_{2}} a_{l_{i j}} X^{i} Y^{j}
$$

with:

$$
l=1,2 \quad 0 \leq m_{1} \leq n ; \quad 0 \leq m_{2} \leq n ; \quad m_{1}+m_{2} \leq n .
$$

This approach requires Ground Control Points (GCPS) with known locations $(X, Y)$ on the map and easy detections $\left(X^{\prime}, Y^{\prime}\right)$ in the image [7]. The substitution of these coordinates values in equations (1) for each GCP permits to obtain polynomial coefficients $a_{l i j^{\prime}}$ In this way (1) can be applied to the whole image so to obtain, for each pixel, the new location in the cartographic plane. The order $n$ of the polynomial functions establishes the minimum number of GCPs that can be easily calculated using the formula:

$$
N_{\min _{G C P S}}=\frac{(n+1) \cdot(n+2)}{2}
$$

To increase positional accuracy of the resulting image, a greater number of GCPs regularly distributed is chosen: in this way the differences between map coordinates and corrected image coordinates for the same features tend to be reduced. Generally, an iterative least-square adjustment process is applied when more GCPs than the minimum number required by the model are used [8].

To evaluate positional accuracy, errors not only for GCPs but also for other points named Check Points (CPS) are considered [9], [10].

Polynomial Functions are not appropriate for rectification of high resolution satellite images: good results are achieved only when the images are acquired with near-nadir viewing angles over a flat terrain [8]. In alternative two main categories of approaches are useful, named parametric and non-parametric methods. The first ones are based on rigorous models that use viewing geometry of the sensor [11]. Non-parametric methods are independent of the sensor characteristics as well as the acquisition mode: relationship between image coordinates (X', Y') and 3D object coordinates $(\mathrm{X}, \mathrm{Y}, \mathrm{Z})$ is typically defined using 3D Rational Polynomial Functions (RPFs) [12], [13]. Particularly:

$$
X^{\prime}=\frac{P_{1}^{n}(X, Y, Z)}{P_{2}^{n}(X, Y, Z)} Y^{\prime}=\frac{P_{3}^{n}(X, Y, Z)}{P_{4}^{n}(X, Y, Z)}
$$

where $P_{1}^{n}, P_{2}{ }^{n}, P_{3}{ }^{n}, P_{4}^{n}$ are usually cubic polynomials (corresponding to 20 coefficients).

For consequence a generic polynomial can be expressed as:

$$
P_{l}^{n}(X, Y, Z)=\sum_{i=0}^{m_{1}} \sum_{j=0}^{m_{2}} \sum_{k=0}^{m_{3}} a_{l_{i j k}} X^{i} Y^{j} Z^{k}
$$

where:

$$
\begin{aligned}
& n=3 \\
& l=1,2,3,4 \\
& 0 \leq m_{1} \leq 3 \\
& 0 \leq m_{2} \leq 3 \\
& 0 \leq m_{3} \leq 3 ; \\
& m_{1}+m_{2}+m_{3} \leq 3
\end{aligned}
$$

Because in the polynomials $P_{2}^{n}$ and $P_{4}{ }^{n}$ the first terms $\left(\mathrm{a}_{2000}\right.$ and $\mathrm{a}_{4000}$ ) are assumed equal to 1,78 coefficients are present in (5) for $n=3$. Their values are calculated by the data provider, considering the position of the satellite at the time of image acquisition and included in RPCs (Rational Polynomial Coefficients) file.

However, they can be calculated using GCPs like in the case above described for Polynomial Functions: at least 39 of them are necessary; when more GCPs than the minimum are introduced, an iterative least-square adjustment process is usually carried out.

The accuracy of the results depends from the number and the distribution of GCPs: several GCPs (more than 39) with a regular distribution both planimetric and altimetric contribute to high quality of results [5], [6], [14]. DEM (Digital Elevation Model) of the whole area is also required for RPFs; consequently in this case the rectification is usually called ortho-rectification.

To carry out geometric corrections of satellite images, GCPs as well as CPs cartographic co-ordinates can be obtained from different sources, such as GPS, air photo surveys, paper or digital maps, GIS, ortho-rectified photos or images, chip data base, etc. depending of the requested accuracy of the input/output data [8].

Applying PFs as well as RPFs to the original image, a matrix of "empty" cells is computed: to calculate the radiometric value (Digital number, DN) to be assigned to each pixel, a resampling method, such as nearest neighbor, bilinear interpolation or cubic convolution, is used [15], [16], [17].

Because resampling functions transform the DNs and then alter the radiometry of the original image, problems may be encountered in subsequent spectral signature or pattern recognition analysis; consequently, any process based on the image radiometry should be performed before using 
interpolation or convolution algorithms [8].

\section{Data and Applications}

IKONOS imagery (Fig. 1) concerning an area in Campania Region (Italy) was considered to compare different methods for rectification; this zone presents interesting environmental as well as anthropic aspects and is included in the National Park of Cilento and Vallo di Diano, a protected area of 181,048 hectares that was established in 1995 [18]. The coast and the orographic profile of the considered area are rugged, with few flat areas around the rivers. If geometrically corrected with adequate positional accuracy, this imagery (such as others with high resolution) can support environmental and territorial studies, i.e. urban growth, change detection, vegetation monitoring, etc.

The dataset was supplied by the provider as CARTERRA GEO product, that means already rectified to a surface with a constant height difference against the earth ellipsoid (low accuracy).

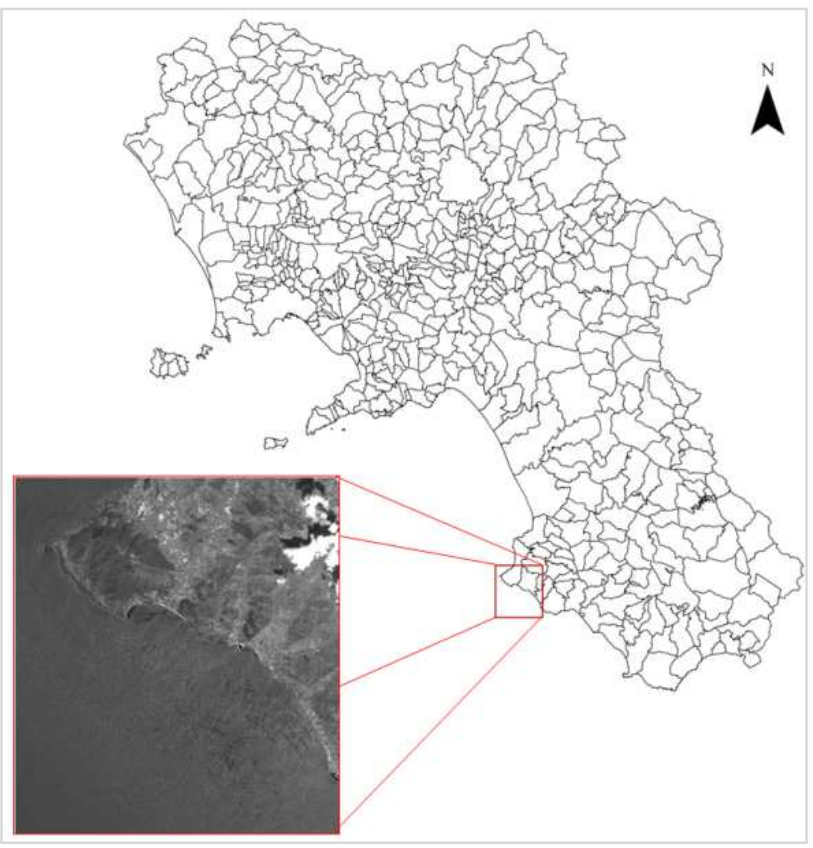

Figure 1. IKONOS scene and its location in Campania Region.

Rectification processes were conducted on the panchromatic image (higher resolution) using PCI Geomatica OrthoEngine Version 2012 in three different ways:

- Polynomial functions;

- Rational Polynomial Functions with original RPCs;

- Rational Polynomial Functions without original RPCs.

GCPs and CPs planimetric coordinates in UTM-WGS84 were derived from ortho-photos of Campania Region with 1 meter resolution (nominal scale: 1:10,000), while elevations were obtained by DEM of the area (cell size: $20 \mathrm{~m}$ ).

The positional accuracy of the original panchromatic image was tested using $20 \mathrm{CPs}$. Table 1 shows statistic values (minimum, maximum, mean, standard deviation, RMS) of errors in these CPs.
Table 1. Residuals (in meters) obtained for CPS in the original IKONOS panchromatic image

\begin{tabular}{llllll}
\hline Original Image & Min & Max & Mean & St. dev. & RMS \\
\hline CPs: 20 & 2.908 & 112.009 & 40.701 & 31.328 & 51.361 \\
\hline
\end{tabular}

To evaluate the accuracy of each rectification process, the residuals obtained for GCPs and CPs in XY direction were calculated. Statistic values of these errors are reported in tables 2, 3 and 4. Particularly the following approaches were considered: PFs of the $4^{\text {th }}$ and $5^{\text {th }}$ orders using 50,60 and 70 GCPs; RPFs with original RPCs using 0, 5, 10, 15, 20, 30, 40, 50,60 and 70 GCPs; RPFs without RPCs of the provider using 50, 60 and 70 GCPs.

For all situations $20 \mathrm{CPs}$ were introduced; a regular (planimetric and altimetric) distribution of GCPs was observed for each combination of them.

Table 2. Residuals (in meters) obtained for GCPs and CPs by using Polynomial Functions.

\begin{tabular}{|c|c|c|c|c|c|c|}
\hline \multicolumn{2}{|c|}{ Polynomial Functions } & \multirow{2}{*}{$\begin{array}{l}\text { Min } \\
0.755\end{array}$} & \multirow{2}{*}{$\begin{array}{l}\text { Max } \\
61.028\end{array}$} & \multirow{2}{*}{$\begin{array}{l}\text { Mean } \\
20.401\end{array}$} & \multirow{2}{*}{$\begin{array}{l}\text { St .dev. } \\
15.937\end{array}$} & \multirow{2}{*}{$\begin{array}{l}\text { RMS } \\
25.888\end{array}$} \\
\hline \multirow{6}{*}{$4^{\text {th }}$ order } & GCPs: 50 & & & & & \\
\hline & CPs:20 & 1.585 & 68.350 & 24.864 & 16.325 & 29.744 \\
\hline & GCPs: 60 & 1.009 & 62.283 & 20.437 & 15.761 & 25.808 \\
\hline & CPs:20 & 4.433 & 70.834 & 25.332 & 16.263 & 30.103 \\
\hline & GCPs: 70 & 0.454 & 78.959 & 21.501 & 15.404 & 26.450 \\
\hline & CPs:20 & 5.174 & 83.348 & 25.514 & 17.658 & 31.029 \\
\hline \multirow{6}{*}{$5^{\text {th }}$ order } & GCPs: 50 & 1.225 & 62.348 & 17.441 & 14.804 & 22.877 \\
\hline & CPs:20 & 1.135 & 60.833 & 20.890 & 16.323 & 26.511 \\
\hline & GCPs: 60 & 0.326 & 62.356 & 18.409 & 15.024 & 23.761 \\
\hline & CPs:20 & 1.060 & 62.672 & 19.437 & 15.442 & 24.825 \\
\hline & GCPs: 70 & 0.790 & 63.780 & 18.540 & 15.212 & 23.982 \\
\hline & CPs:20 & 1.767 & 69.757 & 18.806 & 16.611 & 25.092 \\
\hline
\end{tabular}

Table 3. Residuals (in meters) obtained for GCPS and CPs by using RPFs with RPCs file

\begin{tabular}{llllll}
\hline RPCs & Min & Max & Mean & St. dev. & RMS \\
\hline GCPs:0 & - & - & - & - & - \\
CPs:20 & 11.188 & 16.704 & 13.926 & 1.562 & 14.013 \\
GCPs:5 & 0.863 & 2.132 & 1.718 & 0.447 & 1.776 \\
CPs:20 & 0.720 & 3.360 & 2.134 & 0.848 & 2.297 \\
GCPs:10 & 0.675 & 2.613 & 1.569 & 0.627 & 1.689 \\
CPs:20 & 0.281 & 3.751 & 2.116 & 0.866 & 2.286 \\
GCPs:15 & 0.691 & 2.613 & 1.575 & 0.533 & 1.663 \\
CPs:20 & 0.447 & 3.587 & 2.114 & 0.839 & 2.275 \\
GCPs:20 & 0.618 & 5.119 & 2.169 & 1.013 & 2.394 \\
CPs:20 & 0.582 & 3.482 & 2.154 & 0.895 & 2.333 \\
GCPs:30 & 0.348 & 5.325 & 2.260 & 0.941 & 2.449 \\
CPs:20 & 0.641 & 3.668 & 2.225 & 0.917 & 2.407 \\
GCPs:40 & 0.460 & 9.552 & 2.432 & 1.448 & 2.830 \\
CPs:20 & 0.505 & 3.741 & 2.221 & 0.963 & 2.420 \\
GCPs:50 & 0.390 & 9.873 & 2.446 & 1.527 & 2.884 \\
CPs:20 & 0.700 & 3.408 & 2.164 & 0.871 & 2.332 \\
GCPs:60 & 0.399 & 9.767 & 2.326 & 1.467 & 2.750 \\
CPs:20 & 0.671 & 3.555 & 2.199 & 0.893 & 2.373 \\
GCPs:70 & 0.391 & 9.802 & 2.286 & 1.438 & 2.700 \\
CPs:20 & 0.705 & 3.530 & 2.198 & 0.884 & 2.369 \\
\hline & & & & &
\end{tabular}


Table 4. Residuals (in meters) obtained for GCPs and CPs by using RPFs without RPCs file.

\begin{tabular}{llllll}
\hline No RPCs & Min & Max & Mean & St. dev. & RMS \\
\hline GCPs: 50 & 0.105 & 2.504 & 1.267 & 0.613 & 1.408 \\
CPs:20 & 0.181 & 3.632 & 1.468 & 0.860 & 1.702 \\
GCPs: 60 & 0.124 & 2.429 & 1.210 & 0.590 & 1.347 \\
CPs:20 & 0.453 & 3.235 & 1.428 & 0.818 & 1.646 \\
GCPs: 70 & 0.081 & 2.317 & 1.186 & 0.579 & 1.319 \\
CPs:20 & 0.271 & 3.177 & 1.382 & 0.853 & 1.624 \\
\hline
\end{tabular}

An example of GCP identification is reported in Fig. 2 (location in ortho-photo) and Fig. 3 (location in IKONOS image).

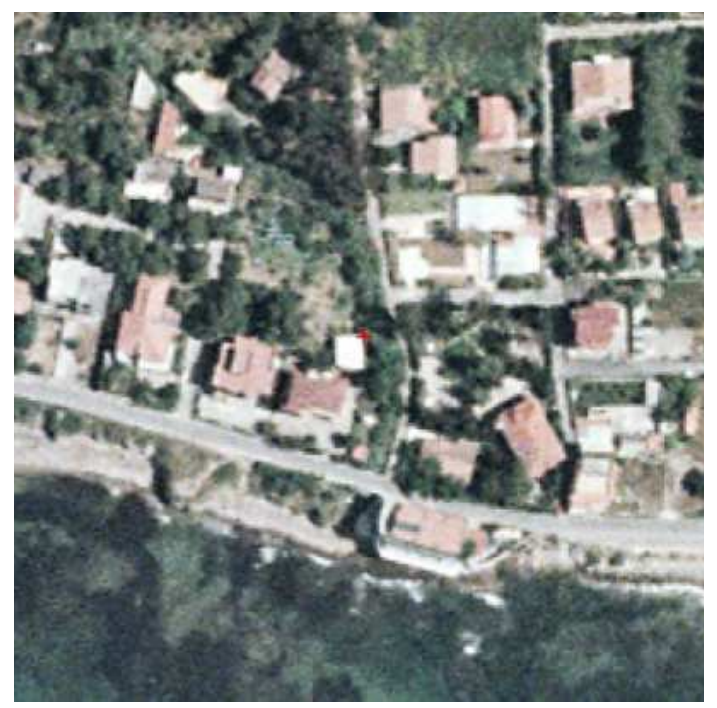

Figure 2. An example of identification of GCP in ortho-photo.

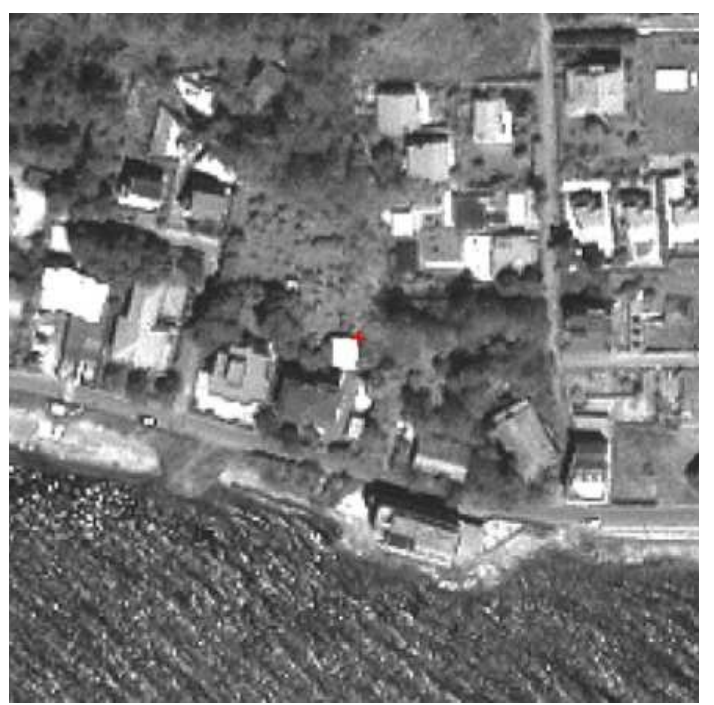

Figure 3. Identification of corresponding GCP in the IKONOS image.

Examples of use datasets of GCPs (50,60 and 70) and CPs (20) for the applications of PFs and RPFs (with as well as without RPCs) are reported in Fig. 4, 5 and 6.

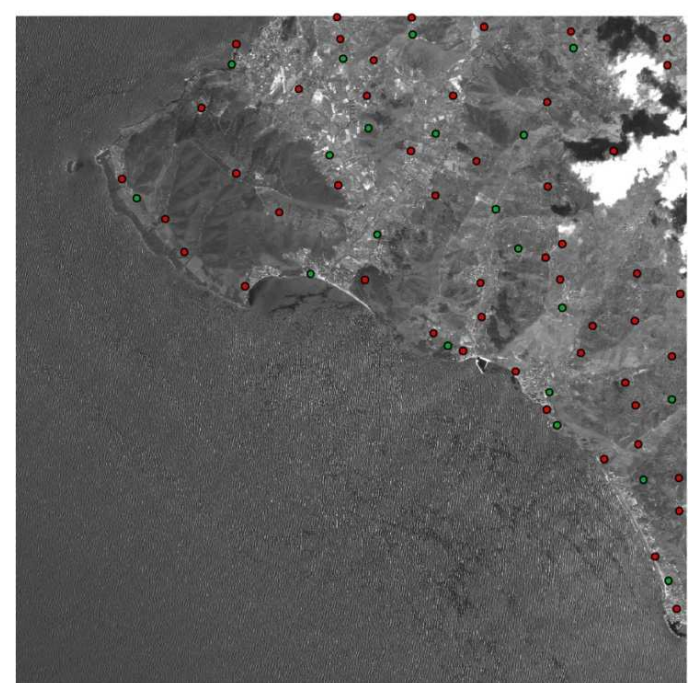

Figure 4. The datasets of GCPS (50, in red) and CPS (20, in green) for the applications of PFs and RPFs.

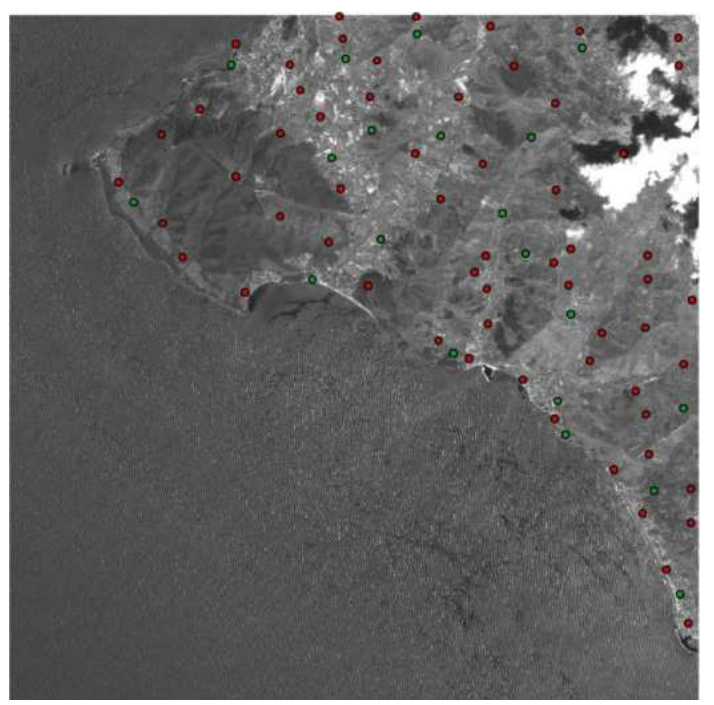

Figure 5. The datasets of GCPS (60, in red) and CPS (20, in green) for the applications of $P F$ s and RPFs.

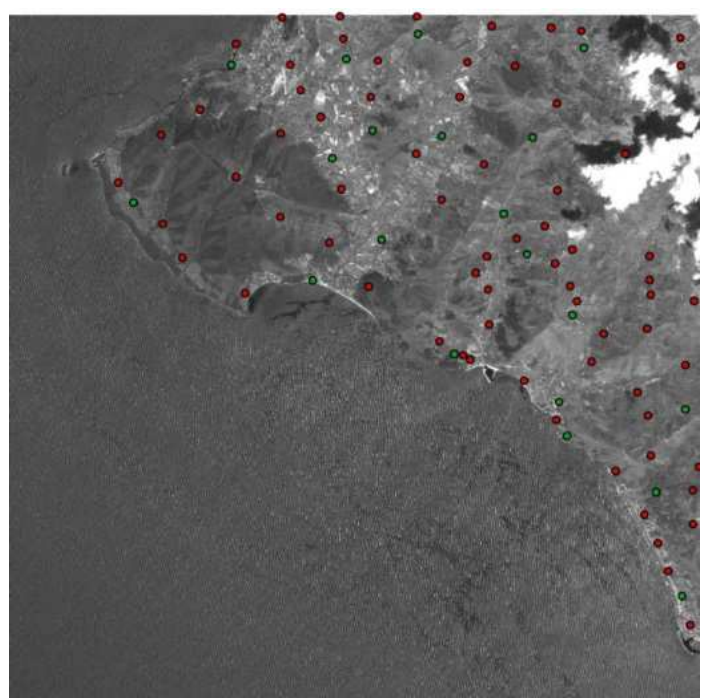

Figure 6. The datasets of GCPs (70, in red) and CPS (20, in green) for the applications of PFs and RPFs. 


\section{Conclusions}

The tests presented in this paper for IKONOS data confirm that PFs are inadequate for high resolution satellite images also when a great number of GCPs is used.

RPFs supply accurate results both with or without the use of RPCs file by the provider: in the first case a lower number of GCPs permits to limit RMS for Ground Control and Check Points; in the second case more accurate results can be achieved, nevertheless several GCPs are necessary (at least 39, but a greater number is suitable). Using RPCs file, RPFs can be applied also without GCPs, but results present considerable positional errors; a little number of GCPs is sufficient to reduce significantly the residuals between the required coordinates and obtained ones. RPFs need 3D information: $\mathrm{X}$, $\mathrm{Y}, \mathrm{Z}$ coordinates must be known for GCPs and DEM of the entire scene is indispensable. However the accuracy of the heights can be lower than that of planimetric coordinates: in the tests, cell size $20 \mathrm{~m}$ for DEM results adequate to obtain acceptable results for image rectification.

Aerial ortho-photos with the same resolution (or better) of the panchromatic image to be rectified can be considered a valid support to obtain coordinates of GCPs and CPs.

\section{Acknowledgements}

This paper synthesizes results of works performed within the project PRIN 2010-11 financed by MIUR (Ministero dell'Istruzione, dell'Università e della Ricerca) - Italy and developed at the University of Naples "Parthenope" (Coordinator: Prof. Raffaele Santamaria).

\section{References}

[1] Apollo Mapping - The Image Hunters, Overview \& History IKONOS specifications, 2014. https://apollomapping.com/imagery/high-resolution-imagery/i konos. Latest access 09/05/2014.

[2] G. Dial, H. Bowen, F. Gerlach, J. Grodecki, R. Oleszczuk, "IKONOS satellite, imagery, and products," Remote sensing of Environment, vol. 88, Issue 1, 2003, pp. 23-36.

[3] M. Herold, J. Scepan, A. Müller and S. Günther, "Object oriented mapping and analysis of urban land use/cover using IKONOS data," Proceedings of $22^{\text {nd }}$ EARSEL Symposium "Geoinformation for European-wide integration", Prague, June 2002.

[4] I. Z. Gitas, C. G. Karydas and G. V. Kazakis, "Land cover mapping of Mediterranean landscapes, using SPOT4 Xi and IKONOS imagery - Preliminary investigation," Options Mediterraneennes, Series B - n 46, 2003, pp. 27-41.

[5] M. Basile Giannini, C. Parente, P. Maglione and R. Santamaria, "Cartography and Remote sensing for coastal erosion analysis," Proceedings of Coastal Process II, 27-29 April, 2011, Naples, WIT Transactions on Ecology and the Environment, Editors: G Benassai, C.A. Brebbia, and G. Rodriguez, WIT Press, ISBN: 978-1-84564-534-2, 2011, pp. 65-76.
[6] A. Errico, F. Guastaferro, C. Parente and R. Santamaria, "Applications on geometric correction of different resolution satellite images," in Proceedings of the IEEE GOLD Conference 2010, Livorno, Italy, 2010, on-line available: http://ieee.uniparthenope.it/chapter/_private/proc10/29.pdf. Latest access 09/05/2014.

[7] E.P. Baltsavias, "Geometric transformations and registration of images, orthoimage generation and mosaicking," Institute of Geodesy and Photogrammetry, ETHZ Zurich, 2000, on-line available:

http://e-collection.library.ethz.ch/eserv/eth:26968/eth-26968-0 1.pdfAsearch $=\% 22$ (keywords en:GEODESY) $\% 22$. Latest access 09/05/2014.

[8] T. Toutin, "Review Article: Geometric processing of remote sensing images: models, algorithms and methods," International Journal of Remote Sensing, vol. 25, Issue 10, 2004, pp. 1893-1924.

[9] E. A. Raabe and R. P. Stump, “ Image processing methods: Procedures in selection, registration, normalization and enhancement of satellite imagery in coastal wetlands," Open-File Report 97-287, USGS, St. Petersburg, FL, 1997.

[10] R. D. Eastman, J. Le Moigne and N. S. Netanyahu, "Research issues in image registration for remote sensing," Computer Vision and Pattern Recognition, 2007. CVPR'07. IEEE Conference on, 2007, pp. 1-8.

[11] I. Parcharidis, M. Foumelis, E. Papageorgiou, M. Segou and V. Sakkas, "Orthorectification and assessment of QuickBird imagery using D-GPS measurements over Paros urban area," in Proceedings of the International Society for Photogrammetry and Remote Sensing 2005 Joint Conference, 2005.

[12] I. Dowman and J. T. Dolloff, "An evaluation of rational functions for photogrammetric restitution," International Archives of Photogrammetry and Remote Sensing 33. B3/1; PART 3, 2000, pp. 254-266.

[13] C. V. Tao and Y. Hu, "A comprehensive study of the rational function model for photogrammetric processing," Photogrammetric Engineering and Remote Sensing 67.12, 2001, pp. 1347-1358.

[14] F. Guastaferro, P. Maglione and C. Parente, "Rectification of Spot 5 satellite imagery for Marine Geographic Information Systems," in Proceedings of ARSA - Advanced Research in Scientific Areas, Virtual Conference, 2012, pp. 1649-1653.

[15] R. C. Gonzalez and R. E. Woods, "Digital image processing," Pearson Prentice Hall, Upper Saddle River, New Jersey 07458, 2002, pp. 65-68.

[16] N. Levin, "Fundamentals of remote sensing," 1st Hydrographic Data Management Course, IMO - International Maritime Academy, Trieste, 1999, pp. 85-86.

[17] J. B. Campbell and R. H. Wynne, "Introduction to Remote Sensing - Fifth Edition," THE GUILFORD PRESS, New York, 2011, pp. 321-324.

[18] Parco Nazionale del Cilento, Vallo di Diano e Alburni, Area Protetta, 2014. http://www.parks.it/parco.nazionale.cilento/par.php, Latest access 09/05/2014. 